Recherches en didactique des langues et des cultures

Les cahiers de l'Acedle

8-1| 2011

Les langues tout au long de la vie: Permanences et évolutions en didactique des langues

\title{
Se former et se transformer par la pratique d'une langue nouvelle en contexte professionnel
}

Michèle Levacic-Burkhardt

\section{(2) OpenEdition \\ Journals}

Édition électronique

URL : http://journals.openedition.org/rdlc/2265

DOI : $10.4000 /$ rdlc. 2265

ISSN : 1958-5772

Éditeur

ACEDLE

Référence électronique

Michèle Levacic-Burkhardt, « Se former et se transformer par la pratique d'une langue nouvelle en contexte professionnel », Recherches en didactique des langues et des cultures [En ligne], 8-1 | 2011, mis en ligne le 28 août 2017, consulté le 03 mai 2019. URL : http://journals.openedition.org/rdlc/2265 DOI : $10.4000 /$ rdlc. 2265

Ce document a été généré automatiquement le 3 mai 2019.

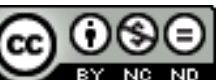

Recherches en didactique des langues et des cultures is licensed under a Creative Commons AttributionNonCommercial-NoDerivatives 4.0 International License 


\title{
Se former et se transformer par la pratique d'une langue nouvelle en contexte professionnel
}

\author{
Michèle Levacic-Burkhardt
}

\section{Introduction}

1 Les expressions telles que "éducation permanente"1, "apprentissage tout au long de la vie"2, " adaptation des compétences", "pluralité des langues et des cultures" jalonnent les discours relatifs à l'éducation et à la formation des adultes. Leur présence marque une certaine volonté politique de répondre aux besoins croissants des technologies, de l'économie, d'un monde en perpétuelle mutation et de continuellement mettre à jour les connaissances et les compétences de chacun à toutes les étapes de la vie en valorisant toutes les formes d'apprentissage. Ces notions évoquent trois thèmes indissociables :

- celui de la formation de l'individu comme un processus long et sans fin ;

- celui de la construction d'un répertoire verbal diversifié ;

- celui de la reconnaissance (Ricœur, 2004) qui implique un certain regard de l'autre sur soi et par voie de conséquence la tendance chez le sujet en quête de reconnaissance, à adopter une attitude qui vise à s'inspirer d'un "modèle", à se rapprocher d'un comportement.

2 Les migrations des populations imposées par la mondialisation obligent les individus à avoir d'autres approches dans le travail, dans la relation aux autres et à développer une qualité de flexibilité pour s'adapter à un environnement en constante mutation.

Comment, en effet, ne pas associer ces trois thématiques quand il s'agit d'avoir un parcours professionnel de migrant et, de fait, d'être confronté à des langues étrangères ? Nos hommes politiques évoquent souvent "le bien vivre ensemble" (Le Monde du 22 avril 2009) dans la gestion des flux migratoires. N'est-il pas la question de faciliter la mobilité, d'encourager le dialogue interculturel et de développer le plurilinguisme? La notion d'éducation permanente n'est-elle pas au cœur de tout le processus ayant motivé la mise en 
place des validations d'acquis de l'expérience? Ces notions sont bien au cœur des débats concernant l'intégration sociale et professionnelle, d'autant plus lorsqu'il s'agit d'évoluer dans un milieu dont la langue n'est pas familière.

4 La formation professionnelle se double alors d'une formation linguistique de première importance car la langue à acquérir est le vecteur des apprentissages ainsi que le médiateur des échanges. Les enjeux sont considérables, notamment pour des professionnels en activité, qui ont le souci de devoir s'intégrer dans une nouvelle communauté.

5 Traiter ces problématiques en synergie revient à considérer l'individu globalement dans ses apprentissages les plus divers, dans son exercice professionnel, et dans ses interactions langagières où l'appropriation et l'utilisation d'un répertoire linguistique varié deviennent une nécessité.

C'est en prenant en compte ces différents aspects que nous avons exploré la notion de "se former et se transformer par la pratique d'une langue nouvelle en contexte professionnel".

7 L'objectif de cette contribution est de livrer un exemple d'acquisition de langue qui relève d'une nécessité professionnelle et qui devient efficiente grâce à une forte motivation guidée par la pression sociale, mais aussi par la volonté de s'immiscer dans une interaction où la démonstration des savoirs et des connaissances relatifs à un métier est indispensable.

\section{2. Éléments de contextualisation et de méthodologie de l'enquête}

8 Pour illustrer cette thématique, nous analysons le cas suivant: celui de migrants allophones, professionnels du secteur médical (médecins diplômés ou en cours de formation) venus en France pour exercer leur métier en milieu hospitalier et ce, sans maîtrise préalable ou en tout cas avec des compétences très partielles de la langue pratiquée sur le terrain. Ce sont des médecins qui ont effectué l'essentiel de leur cursus universitaire dans leur pays et dans leur langue d'origine ${ }^{3}$. Dans leur pays, ils sont déjà diplômés médecins ou en fin de cursus. Lorsqu'ils arrivent en France, ils exercent sous le statut de "faisant fonction d'interne" (FFI).

9 D'après les éléments recueillis auprès de chefs de service responsables de l'accueil et de l'encadrement de ces médecins allophones, les postes de FFI sont attribués à des étrangers lorsqu'ils sont restés vacants après les affectations des internes français. On connaît le problème de la pénurie des médecins en France ${ }^{4}$ (plus ou moins importante selon les régions et les spécialités).

Le système médical considère qu'ils ont les compétences professionnelles requises pour exercer la médecine, en tant qu'interne, mais légalement, ils ne peuvent avoir le même statut. Les compétences linguistiques en français ne sont pas systématiquement vérifiées, pour ces recrutements, comme c'est le cas lorsque des médecins se présentent aux épreuves du concours national des internes à titre étranger 5 .

11 Les motivations des uns et des autres diffèrent. Certains déclarent être venus en France pour une durée courte (de quelques mois à deux années) en vue d'améliorer leur formation, de découvrir d'autres pratiques professionnelles, de justifier de meilleures compétences enrichies par une expérience de la médecine française, réputée dans 
certains pays ; d'autres ont des motivations d'ordre politique ou économique et visent souvent une installation durable en France.

Cette étude s'inscrit dans une recherche de thèse en cours. Elle s'appuie sur l'analyse qualitative d'un corpus oral réalisé à partir d'entretiens semi-directifs menés auprès de ces professionnels allophones mais aussi auprès de chefs de service responsables de leur accueil et de leur encadrement. Ce travail s'appuie pour l'heure sur un ensemble de quatorze entretiens de témoins ainsi répartis : sept Saoudiens, cinq Roumains, un Serbe d'ex-Yougoslavie et un Italien. Ces médecins ont été sollicités par nos soins, de différentes manières : en prenant des contacts dans les écoles où certains d'entre eux scolarisent leur (s) enfant(s), en nous adressant directement à l'hôpital, aux chefs de service qui les encadrent, ou encore en utilisant des relations dans le milieu médical. Les lieux de rencontre étaient fixés de manière pragmatique, dans un bureau de l'hôpital, à la cafétéria de l'hôpital, dans un bar, ou même au domicile de l'enquêteur ou de l'interviewé. Chacun n'a été rencontré qu'à une seule reprise et durant un temps oscillant de trente à soixante minutes. Un guide d'entretien ( $c f$. en annexe) comportant des questions à poser était prévu mais non soumis préalablement à l'entretien.

Tous ne sont pas dotés d'un répertoire linguistique diversifié et font preuve d'une maitrise inégale du français à leur arrivée en France. Bon nombre de ces praticiens ne se sont initiés à la langue française qu'à leur arrivée sur le territoire, en suivant des cours de quelques semaines dans un institut de langues. Aucune prise en charge linguistique officielle (au niveau de l'hôpital ou de la faculté de médecine) n'existe dans la région Centre, terrain où se sont déroulés la plupart des entretiens, à l'exception de deux qui ont eu lieu à Paris. Lorsqu'un dispositif d'apprentissage de la langue française est en place, il relève d'une démarche personnelle du médecin allophone ou de la demande expresse $d u$ chef de service responsable de l'accueil. En effet, il arriva à l'un d'eux d'accompagner personnellement un interne saoudien dans un institut dispensant des cours de français afin qu'il s'y inscrive.

\section{Problématique}

Dans le contexte décrit ci-dessus, où se dessinent des parcours de mobilité géographique diversifiés, où se construisent des répertoires linguistiques pluriels, on assiste à une remise en question des savoirs et des compétences. Nous venons de dire que les médecins FFI étaient dans certains cas déjà titulaires du diplôme de médecin et qu'ils avaient donc déjà eu à assumer des responsabilités professionnelles dans leur pays d'origine. En France, ils sont maintenant FFI, placés sous la tutelle d'un interne français, puis sous celle du chef de service. La moindre action, le moindre écrit (compte rendu, dossier d'entrée du patient) fait l'objet d'un contrôle. Même la secrétaire, chargée de dactylographier un compte rendu enregistré sur dictaphone, demande de revoir la formulation, de corriger la prononciation empêchant une compréhension aisée. Ce qui a été construit par et dans la langue d'origine et dans un milieu différent doit maintenant être justifié, validé, adapté à de nouveaux contextes, professionnel, culturel et linguistique, articulé aux apports issus d'une nouvelle expérience et de la confrontation à l'altérité. Il est important de garder présent à l'esprit que ce sont des professionnels, certes migrants, mais hautement qualifiés.

C'est non seulement un véritable processus de formation, mais c'est aussi un processus de transformation dans la mesure où il y a une remise en cause de toute une pratique 
professionnelle vécue jusque-là, une activité réflexive sur sa propre perception de cette pratique, une réflexion sur le regard qu'y porte autrui et quelquefois une prise de conscience de certains décalages qui nécessitent alors des réajustements. Ainsi, les Saoudiens remarquent la nécessité d'adapter leur comportement face au patient français. Celui-ci demande beaucoup d'explications sur la pathologie qui l'affecte, sur la thérapie envisagée et les conséquences qui en découleront. Le patient saoudien adopterait une posture plus soumise, s'en remettrait à la médecine les yeux fermés, dans une totale confiance, selon le modèle de la relation médecin / patient qualifié de paternaliste ${ }^{6}$. Cette différence d'attitude du patient inscrit nécessairement le médecin dans une autre dimension, dans une autre relation et lui impose un "double exolinguisme" ; il doit être capable de maitriser un langage scientifique puis de transposer ce langage dans un registre vulgarisé pour permettre aux patients d'accéder à la compréhension.

Parmi les nombreuses questions qui émergent, deux font l'objet du présent article :

- Comment se construisent de nouvelles connaissances sur un arrière-plan caractérisé par l'exolinguisme et l'altérité culturelle?

- Comment l'agir professionnel contribue-t-il à développer les compétences langagières dans une nouvelle langue?

17 Considéré comme un expert dans le domaine médical lorsqu'il était dans son pays, le médecin allophone se voit maintenant réduit à un statut de novice, de "faisant fonction d'interne" dans le meilleur des cas, voire d'infirmier dans le pire des cas, il est en situation d'observateur, $d$ '"apprenant" placé sous la tutelle d'un chef de service. Dépourvu de légitimité, il lui faut se reconstruire dans un monde d'incertitude marqué par la rupture avec ce qui était connu, maitrisé et familier. Selon Le Boterf (2005), la compétence professionnelle s'exerce impérativement dans l'action, elle est la résultante de trois facteurs : "le savoir-agir", "le vouloir-agir" et enfin "le pouvoir-agir". Le contexte que nous venons de décrire porte atteinte à la réalisation de l'exercice professionnel dans la mesure où cette dernière composante est entravée. Le médecin allophone n'a pas la légitimité de l'agir, sa compétence n'est pas pleinement reconnue.

\section{Quelques éléments de réponse}

18 Les premières analyses des entretiens, décrits ci-dessus, révèlent que plusieurs phénomènes contribuent à faciliter ces tâches. Ils relèvent du contexte, des attitudes de chacun, des stratégies et du facteur temporel.

\subsection{Connaissances partagées, attitudes, stratégies et actions}

19 La connaissance partagée d'un domaine permet de pénétrer un milieu, de créer plus facilement des liens avec d'autres acteurs présents dans l'interaction. Un élément repéré dans un contexte donné permet d'appréhender tous ses aspects nouveaux: d'autres personnes, une autre langue, une autre pratique, autant d'éléments à considérer sous l'angle de l'altérité. C'est en acceptant non pas de côtoyer mais de s'approprier cette réalité altéritaire que le médecin parvient à faire sien ce qui lui était étranger. En comprenant ce qui lui était inconnu, il devient lui-même Autre. Il subit une certaine transformation de soi, plus ou moins conscientisée. Il sait que sa façon d'agir, de se comporter est différente de celle qu'il aurait dans son pays. Il se construit une identité professionnelle plurielle "selon un processus et des effets d'altération" (Coste, 2009 : 169). 
La (re)construction d'une identité professionnelle et l'intégration au groupe deviennent plus aisées. Bronckart (2001: 143) évoque en d'autres termes cette modification à travers la "notion de personne qui désigne la structure psychique qui s'élabore diachroniquement en chaque individu". Différents courants de recherche relatifs à l'agir professionnel ont inscrit ce concept dans une dimension culturelle articulant le corps dans l'interaction à une communauté de pratiques. Citons les travaux de Lave et Wenger (1991), de Jorro (2006) sur l'agir professionnel des enseignants, ainsi que ceux de Schön (1993) comme références dans le courant de l'approche praxéologique. Pour Schön, l'agir professionnel revêt un savoir-faire observable dans les postures et les agissements spécifiques à tel ou tel professionnel. Chaque corporation professionnelle a ses spécificités. Les professionnels du corps médical sont des praticiens, ce qui signifie a priori, que la pratique joue un rôle d'étayage important dans l'exercice de la médecine. "Les gestes médicaux viennent, dans la période d'adaptation linguistique, compenser les lacunes $d u$ dialogue" (Arpin, 1992 : 107). La pratique permet au sujet en déficit linguistique d'assurer la continuité de son exercice. Pour comprendre ce phénomène, citons ces propos :

Réflexion et pratique sont indissociables. L'une et l'autre se répondent mutuellement et cette réciprocité engendre un mouvement dynamique qui est source de changement et d'ajustement. La dialectique réflexion/pratique assure ainsi une réflexion sur la pratique et une pratique de la réflexion. (Fronteau, 1992 : 176) ${ }^{7}$

En effet dans les curricula de médecine, l'apport théorique se double rapidement d'un apport expérientiel. Les étudiants sont très tôt confrontés à la réalité du terrain. Ce type d'apprentissage basé sur un modèle socio-constructiviste, requiert une forte implication de l'apprenant, quel qu'il soit (étudiant ou sujet plus expert, même en contexte endolingue), dans l'activité qui génère les connaissances. Cette implication se fait par le biais d'interactions, dans la relation à l'autre.

21 Des attitudes telles que l'observation des manières de faire et de dire, l'imitation, l'identification à ses pairs sont des moyens utilisés ; un des médecins rencontrés dit ainsi : "au début, je ne fais rien, je dois observer, écouter le staff et ensuite j'essaie de faire la même chose" (Extrait du corpus des entretiens: un Saoudien). Le médecin allophone observe, s'imprègne de la langue, des façons de faire. En effet,

l'individu s'éprouve lui-même non pas directement, mais seulement en adoptant le point de vue des autres ou du groupe social auquel il appartient. (Baugnet, 1998 : 51)

Aussi, lorsqu'il s'agit de rédiger un compte rendu, le même médecin explique ses stratégies :

je comprends presque $100 \%$ de ce que je lis, mais pour moi, c'est encore difficile de trouver les mots et les phrases, je lis beaucoup de comptes rendus et je choisis les phrases que je comprends très bien et puis j'écris" (Extrait du corpus des entretiens: un Saoudien).

Selon Wenden (1991: 18 cité par Cyr, 1998: 58), il existerait des "stratégies observables" mais également des "stratégies non observables" :

Dans le premier cas, l'apprenant a recours au dictionnaire ou à une demande de clarification sur un fait de langue, il sollicite un autre locuteur pour une répétition, une reformulation ou une aide d'une autre nature. Pour O. Cachet (2009), ces stratégies constituent les "ressources externes" qui regroupent toutes les aides qui relèvent de l'humain et du matériel. Par exemple, un des médecins interrogés, chirurgien viscéral, demande à une infirmière du bloc opératoire de lui dresser une liste des noms de tous les 
instruments chirurgicaux disposés dans la salle. Même si, généralement, les didacticiens privilégient le discours par rapport à une compilation de lexèmes isolés, ce fut une aide considérable pour le témoin en question. La mise en discours des termes mémorisés se fait implicitement dans l'interaction, au moment où ils sont évoqués puis utilisés. La mise en scène de ces ustensiles se substitue à la mise en discours des termes jusque-là méconnus et incompris.

Dans le second cas, lors du recours à des "stratégies non observables", l'apprenant adopte des comportements qui visent à trouver la solution à la difficulté de manière indirecte, jugée plus discrète aussi par un apprenant plus gêné, voire plus complexé. Il agit alors par inférence ou comparaison à une autre langue de son répertoire (souvent l'anglais). En l'occurrence, l'apprenant prend appui sur la fréquence des structures rencontrées et mémorisées grâce à l'imprégnation et à l'attention qu'il porte davantage sur telle ou telle tournure qu'il comprend et qu'il s'approprie au fur et à mesure de son expérience langagière. Lors d'un entretien, un médecin explique ses stratégies lorsqu'il doit rédiger : "Je lis les comptes rendus des médecins et j'utilise les expressions que j'ai l'habitude de voir." (Extrait du corpus : une Roumaine)

D'autres stratégies encore, décrites et classées par Wong et Fillmore (1979 : 209, citée par

Gaonac'h, 1991 : 194) peuvent être remarquées :

- stratégies sociales

S1 : Joignez-vous à un groupe et faites comme si vous compreniez ce qui se dit, même si ce n'est pas le cas.

S2: Donnez l'impression par quelques expressions bien choisies que vous êtes capables de parler la langue.

- stratégies cognitives

C1: Supposez que ce qui se dit est en liaison directe avec la situation ou l'activité en cours. Métastratégie : devinez.

C2: Repérez quelques expressions que vous connaissez et commencez à parler.

C3 : Repérez ce qui se répète dans les expressions que vous connaissez.

C4 : Utilisez au maximum ce que vous avez acquis. 
contournant la difficulté de la langue. C'est l'agir qui suscite la parole et le discours. Pour autant, on ne saurait distinguer de manière très catégorique les processus cognitifs qui relèvent de l'inconscient de ceux qui relèvent du conscient.

Les médecins interviewés, comme nous l'avons déjà mentionné, sont présents sur le terrain. Ils participent aux consultations, aux entretiens avec les patients, aux actes médicaux. C'est dans l'action que l'acquisition de la langue se fait et que les connaissances s'organisent. On comprend alors les recherches de Clot (2001), pour qui le travail est un instrument qui permet une certaine prise de conscience. "[...] le vécu n'est, paradoxalement, pas vécu par celui qui le vit. Il est orienté vers l'objet et le sens de son activité présente et non vers lui-même" (Clot, 2001: 273). Ces propos nous montrent que l'apprentissage d'une langue par le sujet, directement impliqué dans l'interaction sociale se fait d'une certaine façon inconsciemment, et que c'est par la prise de conscience ultérieure de cette expérience lors d'une nouvelle expérience que les notions auxquelles le sujet a été confronté se transforment en savoirs conscientisés et maîtrisés. Bakhtine (1984) avance que comprendre c'est être capable de se confronter à un contexte nouveau. "D'objet vécu hier, [le passé], il est promu au rang de moyen pour vivre la situation présente ou future" (Clot, 2001 : 272).

Un autre élément important dans les modalités d'apprentissage est à prendre en considération, celui de l'autonomie des médecins allophones. En effet, en situation de travail hospitalier, les médecins allophones font remarquer qu'à aucun moment, on ne se soucie du degré de leur compréhension ou de leur incompréhension. Un des chefs de service, responsable de l'encadrement de ces internes allophones, précise le plus simplement du monde, que lorsque la difficulté de s'exprimer entre des membres du personnel médical devient trop "handicapante", un des collègues se charge alors de prendre le relais pour poursuivre la communication (au téléphone, lorsqu'il s'agit par exemple de communiquer des résultats d'analyses biologiques de laboratoire). Le médecin responsable se focalise uniquement sur le résultat, sur l'importance du fait de la transmission de l'information et pas du tout sur les moyens mis en œuvre pour y parvenir, et encore moins sur le sentiment d'échec de l'interne allophone en pareille situation. Il est tout à fait normal et légitime de voir l'importance accordée à la communication de l'information demandée, mais par voie de conséquence, cela renforce la tendance du médecin allophone à ne pas manifester ses difficultés linguistiques. On voit, en effet, d'après ce qu'ils disent en entretien, qu'ils osent rarement manifester leur sentiment d'insécurité linguistique, ils préfèrent paraître bien intégrés dans la communication. Le médecin italien dit : "L'hôpital, c'est pour soigner les patients, pour parler d'eux, je ne peux pas demander à ce moment de m'expliquer la langue, c'est pas le problème des autres." (Extrait du corpus des entretiens). Ils ont le sentiment que la compréhension se fera implicitement, que la question de la langue est plutôt secondaire. Rares sont ceux qui manifestent explicitement des difficultés linguistiques ou même culturelles. Pour ce qui relève du linguistique, les Roumains avancent l'origine latine commune aux langues française et roumaine; les arabophones, souvent anglophones, utilisent des passerelles entre le français et l'anglais.

Plus généralement, tous les éléments extra-verbaux du contexte (imagerie médicale, actes médicaux, état du patient, etc.) contribuent à étayer la compréhension. "Même si d'abord je ne comprends pas les mots, quand je regarde la radio d'un patient j'arrive à savoir le sens de ces mots" (Extrait du corpus : une Roumaine). 

l'appréhension de réussir à s'exprimer, de comprendre peuvent parfois générer des blocages ou au contraire se révéler des éléments stimulants à l'investissement dans les échanges. Il est vrai que cela est vérifiable dans bien des contextes d'apprentissage linguistique. Peut-être qu'en situation professionnelle, les éléments stimulants sont plus présents. Les enjeux étant capitaux, comment ne pas avoir envie de se justifier et ressentir très fort le besoin de faire preuve de professionnalisme lorsqu'un patient-enfant fait la remarque spontanée et "anodine" suivante à un médecin italien pédo-psychiatre : " Mais pourquoi tu parles pas normalement toi ?" (Extrait du corpus : un Italien) et de se faire appeler "docteur spaghetti" ! encore plus compétent pour compenser ce signe distinctif d'altérité, souvent associé, dans l'esprit des gens, à une maitrise insuffisante de la langue (Castellotti, $2008: 33-50$ ), voire même à une compétence professionnelle insuffisante! Une jeune interne Roumaine raconte comment un patient s'interroge sur ses origines: "Mais vous n'êtes vraiment pas comme les Roms qu'on voit dans la rue !..." (Extrait du corpus). Elle dut alors lui faire un petit rappel historique en lui précisant que les Roumains sont des Latins et que les Roms sont issus d'un peuple indien venu s'installer en Roumanie.

37 Le contexte professionnel connu, la motivation de gagner la considération d'autres professionnels, la volonté de ne pas perdre la face sont autant d'éléments qui contribuent à se surpasser pour accéder à une bonne compréhension et à une expression correcte. Il faut aussi prendre en compte un élément non négligeable, celui du temps.

\subsection{Le facteur de temporalité}

38 Un dernier élément, mis en évidence pour le moment et facilitant la tâche des médecins est le facteur temps à mettre en relation avec celui d'expérience. Qui dit "formation tout au long de sa vie", dit expérience et inévitablement temps aussi. La notion de formation contient en elle le facteur de temporalité. En effet, formation et temps sont deux concepts étroitement liés. C'est pourquoi, l'expérience joue un rôle très important dans la formation.

L'étymologie du mot expérience qui vient du latin experiri, signifiant "éprouver" et qui contient la racine per "à travers", induit les notions de passage d'un état à un autre, de changement de soi. On retrouve le radical periri dans periculum, dont le sens est "péril", " danger" (Lacoue-Labarthe, 1997). Les différentes acceptions étymologiques de ce terme nous permettent de mettre en lien l'idée d'épreuve avec celle du risque qu'elle présente. On peut alors avancer que l'expérience consiste en une prise de risque vécue en situation authentique qui peut susciter chez l'individu une sorte d'instinct de survie se traduisant en l'occurrence par de l'investissement personnel, de l'intérêt et de la volonté. La réflexion s'active autour de ce qui est produit et des conséquences qui en découlent. Dewey écrit :

Dire que la pensée intervient en relation avec des situations qui sont toujours en développement et par conséquent inachevées, c'est dire que la pensée se produit quand les choses sont incertaines, douteuses, problématiques (Dewey, 1913, trad.1983 : 182).

Dans le cas présent, la langue non maîtrisée est un facteur déterminant pour engendrer l'entraînement de la réflexion. Cette réflexion s'inscrit dans une continuité ("continuum 
expérientiel" de Kolb, 1984) qui implique de revisiter son expérience à chaque confrontation à une réalité nouvelle pour s'engager activement et consciemment dans des changements afin de rester dynamique dans les processus de formation et de trans formation de soi. L'identité d'un sujet n'est jamais figée, elle se situe dans une dynamique de négociations et de renégociations avec les autres. L'identité d'une personne est façonnée par son entourage. Selon les termes de Ricœur (2005), "l'identité personnelle est marquée par une temporalité qu'on peut dire constitutive. La personne est son histoire." Cette citation nous permet de mener une réflexion sur les effets de l'histoire, constituée en partie de la (des) langue(s) et du (des) contexte(s) dans le(s)quel(s) évoluent les individus.

\section{Les effets de la langue et du milieu sur l'individu allophone}

41 C'est dans une dimension sociale que l'individu, médecin allophone, s'inscrit. Son exercice professionnel connait un grand bouleversement dans la mesure où il doit non seulement se former, mais d'après notre réflexion, aussi se transformer. Bien que toute formation soit, à des degrés divers, une transformation, se former consisterait à devenir conforme à un idéal que l'on se représente, auquel on aspire et se transformer consisterait à tendre vers un au-delà de soi pour se rapprocher des attentes de l'environnement social. Le préfixe "trans" apporte une véritable valeur ajoutée à l'idée de formation. En effet, si l'on en garde l'idée de dépassement, on pourrait analyser cette transformation comme étant le processus du désir de se former, en évoluant vers un "modèle" en présence. L'individu deviendrait alors le siège de tensions entre un "soi idéal" qui correspondrait à "ce que j'aimerais être" et un "soi prescrit" qui correspondrait à "ce que je devrais être" et qui serait constitué de certaines attentes (Philippot, 2003). L'identité d'un individu est composite dans la mesure où elle reflète des éléments contenus chez autrui. "Je ne saurais être moi-même qu'en passant par l'autre ou en m'engageant avec l'autre" (Peperzak, 1995 : 74).

42 "Le moi identifie, et s'identifie à quelque chose de nécessaire à son expression et à son expansion" (Alves-Bernard, 1996 : 108). En l'occurrence, cette expansion révèle le développement de soi, par l'acquisition de la langue du milieu permettant d'exercer la profession médicale, mais aussi l'appropriation des éléments du contexte par le sujet pour être en harmonie et atténuer les traces de décalage et de rupture. Un exemple flagrant peut illustrer le phénomène qui consiste en la transformation de l'individu par le processus d'apprentissage.

43 Un médecin italien, arrivé en France sans maîtrise de la langue française ayant le statut d'interne dans un hôpital français, décrit les relations qu'il entretient avec ses collègues du service. Il est très étonné de la faible intensité des voix des médecins français.

-"À mon arrivée en France, je ne comprenais vraiment pas beaucoup la langue française. Dans les réunions du service, j'étais très attentif pour essayer de comprendre. Déjà que je ne comprenais rien, en plus je n'entendais rien, tout le monde parlait très doucement." (Extrait du corpus : un Italien)

45 -"Un jour, une stagiaire italienne est arrivée dans le service. Elle parlait pas mal le français. Mais, j'étais très mal à l'aise quand elle était là. Elle osait parler très fort, elle criait pour m'appeler. Un peu plus tard, quand je la connaissais mieux, je lui ai dit : tu sais, ici, c'est pas comme chez nous... il 
faut que tu te calmes un peu ...parce que tout le monde va se demander pourquoi tu parles très fort comme ça! " (Extrait du corpus : le même Italien) parviennent-ils à construire de nouvelles connaissances et à se révéler des professionnels efficaces et comment, dans un positionnement inverse de ce qui est habituellement observé $^{10}$ (Levacic-Burkhardt, 2010), leur agir professionnel favorise-t-il l'accès à une nouvelle langue? Forte motivation, immersion en milieu professionnel et action par mimétisme, imitation, conformation ou identification seraient les principales sources de succès de ces médecins. Mais l'élément qui renforce ces possibilités de réussite vient aussi du fait que la formation au métier de médecin s'effectue en grande partie au contact direct de la réalité concrète du terrain, c'est-à-dire dans un cadre contextualisé aux côtés de professionnels expérimentés. Les médecins des hôpitaux sont d'ailleurs appelés des praticiens hospitaliers. Un désir d'apprendre, étroitement imbriqué au vécu social, permettrait de conquérir un monde perçu comme partiellement étranger. Désir d'apprendre, certes, mais dicté par une impérieuse obligation, celle de l'intégration sociale et de l'épanouissement personnel. Même si l'idée d'une nécessaire pression sociale peut paraitre excessive, il faut en reconnaitre quelques vertus en retenant les propos de Dewey, selon lesquels : 
la seule vraie éducation provient de la stimulation des capacités de l'enfant sous la pression des situations sociales dans lesquelles il se trouve (Dewey, 1897, trad. 1995 : 111).

Par ailleurs, nous constatons qu'une certaine aisance dans la maîtrise de la langue est relativement vite acquise. Il s'agit d'un registre de langue plutôt spécialisée (la communication au sein de l'équipe médicale) mais à la fois d'un registre de langue courante (la communication avec les patients). Si l'on considère que la motivation et l'ancrage en situation authentique sont des éléments évidents pour favoriser l'investissement personnel, il semble en tout cas que la didactique des langues ne les déconsidère pas (cf. Cecrl: Cadre Européen Commun de Référence pour les Langues, 2001). Désormais, on parle de "perspective actionnelle"; il ne s'agit plus seulement de communiquer avec l'autre mais d'agir avec lui dans une co-action. On se forme à devenir un acteur social en agissant socialement dans le cours de l'apprentissage car on se prépare à vivre et à travailler, non seulement dans son propre pays mais aussi dans un autre pays avec des locuteurs d'une autre langue que la sienne.

Pour achever ce propos, nous retiendrons la citation suivante, tirée de l'œuvre de Dewey : "Apprendre? Certainement; mais vivre d'abord, et apprendre par la vie et dans la vie" (cité par Alves-Bernard, 1996 : 101), à condition évidemment de "réflexiver" son expérience pour qu'elle se transforme en compétence.

Se former à n'importe quel moment de sa vie ${ }^{11}$, en apprenant un métier et une langue concomitamment requiert de combiner :

- l'affectif qui concerne la personne dans son intimité ;

- le cognitif qui englobe toutes les possibilités d'appréhender les connaissances et de se les (ré)approprier ;

- le social, c'est-à-dire le contexte interactionnel des acquisitions.

Plus spécifiquement, être confronté à l'altérité culturelle et linguistique en contexte professionnel place le sujet apprenant dans des sphères de références différentes, en tension entre stratégies d'adaptation et résistance aux changements. Il doit supporter la sensation d'un recommencement permanent, ce qui semble contraire à l'idée de développement de soi mais qui pourtant s'avère être une source d'enrichissement et de progrès et qui contribue à la réalisation du phénomène de transformation de soi, dans le sens d'un dépassement de soi, d'un mouvement qui projette le soi au-delà de ce qui était attendu et de ce qu'il était capable de faire.

\section{BIBLIOGRAPHIE}

Alves-Bernard, I. (1996). "Des médiations éducatives à l'actualité de John Dewey". In Revue Spirale , Revue de Recherches en éducation, $\mathrm{n}^{\circ}$ 17, pp. 101-118.

Arpin, J. (1992). "L'identité culturelle du clinicien et son rôle dans sa relation avec le patient". In Santé mentale au Québec, vol. 17, n², pp. 99-112. Disponible en ligne. http://id.erudit.org/revue.

Bailly, D. (1997). Didactique de l'anglais (1). Objectifs et contenus de l'enseignement. Paris : Nathan. 
Bakhtine, M. (1984). Esthétique de la création verbale (A. Aucouturier, trad.). Paris : Gallimard.

Baugnet, L. (1998). L'identité sociale. Paris : Dunod.

Bronckart, J.-P. (2001). "S'entendre pour agir et agir pour s'entendre". In Baudoin, J.-M. \& Friedrich, J. (Eds). Théories de l'action et éducation. pp. 133-154. Bruxelles : De Boeck Université.

Cachet, O. (2009). "Professionnalisme des enseignants et complexité : vers une conception dynamique de l'agir". In Lidil n³9. Disponible en ligne. http://lidil.revues.org/index2747.html. Consulté le 23 mars 2011.

Castellotti, V. (2008). "Apprendre le français sans accent? ou à la recherche du professeur idéal perdu". In L'oral en représentation (s), (Eds.) C. Parpette et M-A. Mochet, pp. 33-50. Bruxelles :

E.M.E.

Clot, Y. (2001). "Clinique du travail et action sur soi". In Théories de l'action et éducation, (Eds.) Conseil de l'Europe. (2001). Cadre européen commun de références pour les langues. Paris : Didier. Coste, D. (2009). "Postface Médiation et altérité". In Lidil , n 39. Disponible en ligne. http:// lidil.revues.org/index2752.html. Consulté le 23 mars 2011.

Cyr, P. (1998). Les stratégies d'apprentissage. Paris : CLE International.

Dewey, J. (1897). Mon Credo pédagogique. (Deledalle, trad. 1995). Paris: PUF.

Dewey, J. (1916). Démocratie et éducation (Deledalle, trad., 1983). Lausanne : Edition l'Âge d'Homme.

Fronteau, J. (1992). "La formation n'est pas un prêt-à-penser". In Santé mentale au Québec, vol. 17, $\mathrm{n}^{\circ}$ 2, pp. 171-181. Disponible en ligne. http://id.erudit.org/iderudit/502076ar

Gaonac'h, D. (1991). Théories d'apprentissage et acquisition d'une langue étrangère. Paris : Didier.

Jorro, A. (2006). L'agir professionnel des enseignants. Disponible en ligne. http://halshs.archivesouvertes.fr/halshs-00195900/fr/. Consulté le 12 mars 2010.

Klein, W. (1984). L'acquisition d'une langue étrangère (trad. par Noyau C., 1989). Paris: A. Colin.

Kolb, D. A. (1984). Experiential Learning - Experience as the source of learning and development.

Englewoods Cliffs (NJ) : Prentice-Hall. Disponible sur en ligne. http://

pedagogieuniversitaire.files.wordpress.com/.../questions_kolb.pdf

Krashen, S. (1981). Second language acquisition and second language learning. Oxford: Pergamon.

Lacoue-Mabarthe, Ph. (1997). La poésie comme expérience. Paris : Christian Bourgeois.

Lave, J. \& Wenger, E. (1991). Situated Learning: Legitimate Peripheral Participation. Cambridge : Cambridge University Press.

Le Boterf, G. (2005). Construire les compétences individuelles et collectives. Paris : Éditions d'Organisation.

Levacic-Burkhardt, M. (2010). "L'agir professionnel, facteur d'insertion et d'appropriation linguistique". In Blanchet, P. \& Coste, D. (dir.), Regards critiques sur la notion d"interculturalité". Pour une didactique de la pluralité linguistique et culturelle, Collection "Espaces discursifs", Paris :

l'Harmattan, 2010, p. 115-129.

Moirand, S. (1982). Enseigner à communiquer en langue étrangère. Paris : Hachette. 
Peperzak, A. (1995). "Autrui et moi-même comme autrement autres". In Paul Ricoeur,

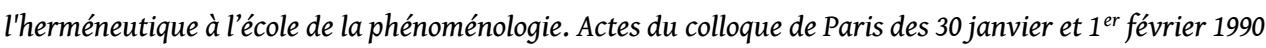
et du colloque de Naples des 7 et 8 mai 1993. Paris : Beauchesne. pp. 73-84.

Philippot, P. (2003). "Les voies du changement personnel". Revue Sciences humaines, hors série n

$\circ$ 40, mars-avril-mai 2003. p. 70.

Ricoeur, P. (1990). Soi-même comme un autre. Paris : Seuil.

Ricoeur, P. (2004). Parcours de la reconnaissance. Paris : Stock.

Ricoeur, P. (2005). " Devenir capable, être reconnu ". Esprit, nº 7, Juillet 2005.

Schön, D. (1993). Le praticien réflexif. À la recherche du savoir caché dans l'agir professionnel. Montréal : Éditions Logiques.

Springer, C. (1996). La didactique des langues face aux défis de la formation des adultes. Paris : Ophrys.

\section{ANNEXES}

Annexe : Guide d'entretien

\section{Introduction à l'entretien :}

Dans le cadre de ma thèse, $\mathrm{j}$ 'étudie comment les individus, amenés pour diverses raisons à migrer, gèrent leurs compétences dans leur exercice professionnel en particulier lors de l'utilisation d'une langue différente de celle dans laquelle ils ont été formés.

Comment un individu parvient-il à exercer une profession dans une langue autre que celle qui a servi à sa formation?

\section{Des origines et des informations personnelles?}

- Pouvez-vous me parler de vos origines? D'où venez-vous? Depuis combien de temps êtes-vous en France? Avez-vous travaillé ailleurs que dans votre pays et ailleurs qu'en France?

- Quel est votre projet professionnel?

- Quelle est votre langue maternelle? (La langue que vous utilisiez dans votre enfance, avec vos parents, vos amis)

- Quelle(s) autre(s) langue(s) parlez-vous?

\section{De la formation scolaire et professionnelle}

- Quel est votre statut à l'hôpital?

- Quelle formation avez-vous eue?

- Quel a été votre parcours scolaire et universitaire?

- Dans quel(s) pays êtes-vous allé à l'école puis à l'université ?

- Quelle (s) langue (s) y parlait-on ? En quelle langue avez-vous passé vos examens?

- Quelle(s) langue(s) avez-vous étudiée(s) ? À partir de quand et combien de temps ?

- Dans votre cursus universitaire avez-vous utilisé d'autres langues? Avez-vous déjà eu des cours d'une quelconque spécialité dans une autre langue? 
- A partir de quand avez-vous commencé à utiliser la langue en cours d'acquisition ou d'apprentissage en dehors du cours de langue ? En d'autres termes, quand avez-vous commencé à utiliser la langue pour construire des connaissances médicales?

\section{De l'activité professionnelle}

- Dans quelle langue travaillez-vous au quotidien?

- Avez-vous eu l'occasion d'utiliser une autre langue dans le cadre de votre travail ?

- Est-ce que vous utilisez plus l'oral ou l'écrit?

- Quel type d'écrit lisez-vous? Produisez-vous? (Contenus et destinataires)

- Cela vous demande-t-il des efforts particuliers? Lesquels?

\section{Des rapports : langues /individu et langues / compétences}

- Dans quel(s) contexte(s) utilisez-vous les langues que vous connaissez ?

- Dans quelle(s) langue(s) êtes-vous le plus à l'aise ? Comment l'expliquez-vous?

- Avez-vous l'impression de réserver les langues de votre répertoire à des domaines spécifiques (sphère sociale, professionnelle, familiale)?

- Quelle(s) difficultés avez-vous rencontrées dans les débuts de votre activité professionnelle (parler au quotidien, exprimer des idées, faire part des connaissances liées à votre spécialité)?

- Comment y avez-vous remédié?

- Avez-vous ressenti le besoin d'une nouvelle formation ou d'un complément de formation dans votre domaine?

- Avez-vous eu l'impression de faire de la traduction lors du passage au français ou bien s'agissait-il plutôt de nouvelles connaissances que vous construisiez ?

- Ces connaissances ou ces compétences se modifiaient-elles lors du passage à l'autre langue ? Est-ce que vous avez le sentiment de dire la même chose que dans votre langue?

- Avez-vous eu l'impression de modifier certaines façons de penser lors de ce passage ?

- Avez-vous lu beaucoup d'ouvrages relatifs à la médecine en langue française ?

- Avez-vous l'impression que vos compétences professionnelles évoluent ou se modifient parce que vous travaillez dans une autre langue (ceci indépendamment de votre expérience)?

- Notez-vous d'importantes différences dans la façon de travailler pratiquée en France par rapport à celle de votre pays ? Dans la relation aux patients ?

- Est-ce qu'il vous paraît difficile de travailler dans une langue différente de celle dans laquelle on a étudié?

- Si vous deviez donner des conseils à quelqu'un qui s'apprêterait à vivre la même expérience que la vôtre, que lui conseilleriez-vous ? Le mettriez-vous en garde contre quelque chose de particulier?

- Pouvez-vous expliquer comment vous réussissez à exploiter vos connaissances construites dans votre langue d'origine en français? 
- Est-ce que vous vous êtes déjà senti(e) démuni(e) dans vos débuts, un peu comme on peut l'être dans une situation de communication ordinaire, avec un étranger?

- Les changements que vous avez vécus, sur les plans géographique, linguistique, culturel, vous donnent-ils l'impression d'être quelqu'un d'autre, de ne plus être vous-même, surtout lorsque vous vous exprimez en français?

- Au contraire, avez-vous l'impression de subir une modification plus profonde de votre personne, dans votre for intérieur?

- Pouvez-vous dire que la connaissance et surtout la pratique régulière d'une nouvelle langue entraînent une modification de soi, de son identité ?

- Vous êtes-vous senti(e) revenir en arrière?

- Avez-vous eu l'impression de ne pas être pris(e) comme un(e) véritable professionnel (le) ?

Remarque: Cette série de questions sert de trame à l'entretien. Les questions ne sont pas forcément posées dans cet ordre ni formulées de cette façon (suivant le degré de compréhension du témoin).

\section{NOTES}

1. Éducation permanente est d'ailleurs le nom d'une revue, créée en 1969 par Bertrand Schwartz à Nancy et c'est la première revue francophone de recherche dans le champ de la formation des adultes.

2. Ce concept inspiré par Jacques Delors est apparu pour la première fois dans le Livre Blanc (1993) intitulé "Croissance, compétitivité, emploi : les défis et les pistes pour entrer dans le 21ème siècle."

3. Il faut comprendre "langue d'origine" au sens de langue première et / ou de scolarisation. $C^{\prime}$ 'est le cas notamment des individus issus de pays arabes, arabophones dans la sphère familiale et dans les échanges de la vie quotidienne mais anglophones dans le cursus universitaire.

4. http://www.lepoint.fr/sante/demographie-vers-une-penurie-demedecins-24-11-2010-1266207_40.php

5. Cf. Décret n $90-97$ du 25 janvier 1990

6. Dans ce modèle, le médecin est considéré comme le garant de l'intérêt du patient, comme celui qui sait et qui est objectif. À l'opposé, le modèle délibératif établit une sorte de contrat thérapeutique qui impose au médecin d'expliquer toutes les modalités du traitement à son patient ainsi que son consentement.

7. J. Fronteau est psychologue et formateur en relations interculturelles à Montréal. Il a travaillé sur le processus migratoire en distinguant la problématique des réfugiés de celle des immigrants. L'intérêt double de cette citation tient au lien que l'auteur fait entre pratique et réflexion, même s'il ne fait pas référence aux praticiens de la médecine. En effet, nous pouvons rapprocher ces deux notions pour deux raisons. D'une part, la pratique, incontournable en médecine, vient relayer l'expression et la compréhension et d'autre part, la réflexion permet au sujet, "défaillant" sur le plan linguistique de se positionner de manière réflexive par rapport à son vécu, d'avoir l'impression de réapprendre ce qu'il savait déjà. Fronteau prend un exemple simple : il demande à un droitier d'écrire son prénom de la main droite, puis dans un second temps, il lui demande de le réécrire de la main gauche. Cette fois, l'individu qui se livre à cet exercice doit s'appliquer, réfléchir et réapprendre en quelque sorte un geste qui était pourtant, jusque-là maîtrisé et réalisé automatiquement. Il en est de même pour les tâches réalisées dans un contexte 
exolingue ; le migrant doit sans cesse revoir ce qu'il avait l'habitude de faire et d'exprimer dans sa langue première pour le transposer en l'adaptant aux exigences de la nouvelle langue et de la nouvelle culture.

8. Les termes "monitor theory" sont traduits par "théorie du contrôle" par Klein (1984, trad. française 1989: 44). Cette théorie postule que l'adulte a deux systèmes indépendants pour développer ses connaissances en langue étrangère, à savoir, "l'apprentissage conscient de la langue" ("conscious language learning") et l'"acquisition subconsciente de la langue" ("subconscious language learning"). Selon cette théorie, lorsque le sujet apprend une langue, il est attentif à, et donc conscient de la forme linguistique des énoncés qu'il produit, mais ce n'est pas le cas dans l'"acquisition" qui est marquée par l'absence d'une telle conscience chez le sujet.

9. Krashen a distingué les processus d'acquisition (spécifiquement informel, observé en milieu naturel, non guidé) et d'apprentissage (spécifiquement formel, observé en milieu institutionnel et guidé) en minimisant la réflexion sur la langue qui n'interviendrait qu'à certains moments de la production langagière. On lui opposera la nécessité d'accorder une importance prépondérante à l'activité métalinguistique du sujet apprenant une langue. Le temps d'exposition à la langue 2 n'est pas toujours suffisant pour permettre une acquisition optimale des structures syntaxiques et doit être compensé par une activité cognitive sur la langue (Bailly, 1997). Springer (1996) exprime un point de vue semblable et tout aussi critique en avançant que Krashen devrait considérer le Monitor non pas comme "un appendice" mais comme "une pièce maîtresse de la construction des savoirs". Malgré ces critiques, nous maintenons la référence à Krashen, dans le cas présent, car il s'agit précisément d'une immersion totale dans la langue 2 et sa théorie garde toute sa pertinence.

10. On a tendance à considérer, dans un ordre logique des choses, que la connaissance de la langue doit précéder l'insertion professionnelle, or ici, nous construisons progressivement la preuve que le contraire est possible.

11. On observe en effet les mêmes comportements dans l'enfance en milieu scolaire, chez des élèves nouvellement arrivés en France (Enaf, Bulletin officiel spécial de l'Éducation nationale, $\mathrm{n}^{\circ} 10$ du 25 avril 2002) qui sont scolarisés dans leur classe d'âge, malgré un degré de francophonie très bas ou inexistant. Ils doivent néanmoins s'intégrer et apprendre leur "métier d'élève" en exploitant les savoirs construits dans leur langue d'origine.

\section{RÉSUMÉS}

Se former lors de parcours migratoires impose de fait l'adaptation à d'autres cultures et l'appropriation d'une ou plusieurs langues. Pourtant, exercer sa profession dans une autre langue et dans un autre pays suscite bien d'autres processus cognitifs. Nous proposons ici l'exemple de médecins allophones exerçant leurs compétences professionnelles en milieu hospitalier français malgré une maîtrise partielle de la langue qui s'y pratique. Les exigences du métier rendent l'acquisition de la langue incontournable, celle-ci devient efficiente grâce à une forte motivation guidée par la pression sociale, mais aussi par la volonté de s'immiscer dans une interaction où la démonstration des savoirs et des connaissances, relatifs à ses compétences, s'impose pour gagner la reconnaissance de ses pairs et une certaine légitimité. Appréhender l'inconnu, en s'appropriant les éléments de l'altérité contribue à la construction d'une identité professionnelle plurielle. La "praxis", l'action située des praticiens spécifique au contexte de l'exercice médical, des procédés cognitifs inspirés de l'imitation, une activité réflexive sur ses 
expériences diverses, l'analyse du regard porté par l'autre sur soi, sont autant d'éléments qui facilitent la réalisation de la tâche du médecin allophone.

Educating oneself all life long as a migrant actually involves adapting to other cultures and making one language, or more, one's own. And yet, having to work in a foreign country using a vernacular which is not one's own arouses a number of other cognitive processes. This paper analyses the case of allophone physicians practising in French hospitals without any prior mastery of the language spoken there. The acquisition of the French language is inevitable if they want to practise their job in a French environment and they need a strong motivation. The social pressure, the will to interact with their peers and the necessity to prove a professional knowledge are very heavy. They have to be up to the expectations in order to be legitimized and recognized for their competence. Understanding unknown elements of otherness makes people with a multicultural professional identity. The "praxis", acting in a context characteristic of medical practice, cognitive processes inspired by imitation, a thought about his own experience, all of this contributes to make easier the physician's job.

\section{INDEX}

Keywords : language-appropriation strategies, experience, identification with another individual, education and training, self-transformation, recognition

Mots-clés : stratégies d'appropriation d'une langue, expérience, identification d'une personne à une autre, formation, transformation de soi, reconnaissance

\section{AUTEUR}

\section{MICHĖLE LEVACIC-BURKHARDT}

Michèle Levacic-Burkhardt est doctorante en Sciences du langage à l'Université FrançoisRabelais de Tours. Membre de l'équipe Dynadiv (EA 4246), elle travaille sur la mobilité des ressources cognitives, langagières et professionnelles des médecins allophones en situation de migration. Par ailleurs, elle enseigne le français langue seconde aux élèves nouvellement arrivés en France (Enaf) dans le cadre de l'Éducation nationale. Elle intervient dans la formation des étudiants en Master MEEFA pour enseigner l'ethnologie de la communication. Courriel : $\underline{\text { michelelevacic[at]gmail.com }}$ 